\title{
Diagnostic difficulties and therapeutic choices in intrapancreatic accessory spleen: case reports
}

This article was published in the following Dove Press journal:

Open Access Surgery

18 March 2016

Number of times this article has been viewed

\author{
Marco Massani' \\ Paola Maccatrozzo' \\ Giovanni Morana ${ }^{2}$ \\ Luca Fabris ${ }^{3}$ \\ Cesare Ruffolo' \\ Luca Bonariol' \\ Bruno Pauletti' \\ Nicolò Bassi' \\ 'IV Department of Surgery, Regional \\ Center for HPB Surgery, ${ }^{2}$ Diagnostic \\ and Interventional Radiology, Regional \\ Hospital of Treviso, Treviso, ${ }^{3}$ Molecular \\ Medicine Department, University \\ Hospital, Padua, Italy
}

Correspondence: Marco Massani IV Department of Surgery, Regional Center for HPB Surgery, Regional Hospital of Treviso, Piazza Ospedale, I-31 100 Treviso, Italy Tel +39042232 2450 Email mmassani@ulss.tv.it
Introduction: Accessory spleen has a worldwide prevalence of $10 \%-30 \%$ and is defined as intrapancreatic accessory spleen (IPAS) when it locates within the pancreas. This occasional finding can raise difficulties in differential diagnosis with hypervascular pancreatic lesions, such as pancreatic neuroendocrine tumor.

Presentation of cases: We present five cases in which a mass of the pancreatic tail was found on radiologic investigations. The first case was a young female patient who underwent a distal pancreatectomy because of a mass of the pancreatic tail misdiagnosed as a pancreatic neuroendocrine tumor due to its radiologic features on computed tomography and magnetic resonance imaging (MRI) (small, ovoidal, and well defined). Misdiagnosis also occurred in the second case, in which an 82-year-old woman was referred to our hospital because of a pancreatic mass of uncertain diagnosis. She also underwent an operation, and pathologic examination showed splenic parenchyma. A correct diagnosis was achieved in the remaining three cases that are still under radiologic monitoring.

Discussion: IPAS is a benign entity and therefore does not require surgical treatment. We discuss the best diagnostic options that have recently been experienced, focusing on diffusion-weighted and superparamagnetic iron oxide MRI, which in our experience seem to be the safest and most easily accessible diagnostic tools.

Conclusion: We suggest that a multidisciplinary approach should guide the diagnosis. When a pancreatic mass with specific features (round, ovoid, and well defined) is detected by computed tomography or MRI, an IPAS should be suspected.

Keywords: intrapancreatic accessory spleen, superparamagnetic iron oxide, diffusion-weighted images, neuroendocrine tumor, neoplasm

\section{Introduction}

Accessory spleen is a relatively common finding, being identified in $10 \%-30 \%$ of autopsies. ${ }^{1-4}$ Accessory spleens are usually found in the splenic hilum ( $80 \%$ of cases), followed by the pancreas (17\%), the splenic ligament, the adipose capsule of the kidneys, the ileum wall, the mesentery, the omentum majus, the female adnexa, and the scrotum. ${ }^{2}$

Physicians find the diagnosis of intrapancreatic accessory spleen (IPAS) somehow challenging, since it poses no clinical threat and therefore does not require surgical procedures.

Generally, pancreatic masses of uncertain origin are removed surgically, issuing no therapeutic effects, increasing risks for patients, and thus giving rise to ethical and legal issues. ${ }^{3}$

Differential diagnosis includes pancreatic ductal adenocarcinoma, pancreatic neuroendocrine tumor (pNET), solid pseudopapillary tumors, and metastases. ${ }^{4}$ 
We present the cases of five patients with radiologic evidence of pancreatic masses; two of them underwent surgical procedures and microscopic examinations revealed IPAS. The other three underwent periodic radiologic examinations, which confirmed the benignity of the lesions.

Our aim is to address the issue of mistaken diagnosis and to discuss the best diagnostic options, focusing on noninvasive radiologic methods that have recently been experienced. Written informed consent was previously acquired from the patients, and work was carried out according to ethical regulations. The Ethics Committee for Clinic Experimentation of the Regional Hospital of Treviso approved the study.

\section{Case report I}

A pancreatic mass was found in a 37-year-old woman undergoing some routine follow-up investigations for a previously resected cervix neoplasm.

Multidetector-row computed tomography (MDCT) revealed a $1.8 \mathrm{~cm}$ focal lesion in the pancreatic tail on contrast phase. The lesion showed a peripheral arterial enhancement (Figure 1A) with a central hypodense area, unenhancing in both the arterial and venous phases (Figure 1B). Magnetic resonance imaging (MRI) confirmed the presence of a round and well-defined nodule within the pancreatic tail. The nodule showed a solid peripheral pattern, while the central hypodense area (seen on MDCT) appeared hypointense on T2-weighted and isointense on T1-weighted images (Figure 1C and D). Gadolinium (Gd)-enhanced MRI confirmed the enhancement of the solid component in the arterial phase, without significant washout in the venous phase (Figure 1E and $\mathrm{F}$ ), while the central core did not show significant enhancement. No relevant adenopathy was found.

These radiologic findings suggested a pNET with a hemorrhagic central area. Laboratory values were as follows: hydroxyindoleacetic acid $2.2 \mathrm{mg} / \mathrm{L}, 5 \mathrm{OH}$-indole acetic acid (U/24 h) $4.8 \mathrm{mg} / 24 \mathrm{~h}$, neuro-specific enolase $7.7 \mathrm{ng} / \mathrm{mL}$. Positron emission tomography showed no pathological metabolism. A distal pancreatectomy was eventually performed.

The surgical specimen contained a smooth, dark red nodule of $\sim 1.5 \mathrm{~cm}$ (Figure $1 \mathrm{G}$ ). The mass was later diagnosed as an IPAS (Figure 1H). Postoperative recovery was uneventful.

\section{Case report 2}

An 82-year-old woman was referred to our hospital because of a mass in her pancreatic tail discovered on a computed tomography (CT) scan. She had previously undergone splenectomy for Werlhof's disease and had other relevant comorbidities such as diabetes, atrial fibrillation, ischemic cardiomyopathy, rheumatoid arthritis, and obesity. The patient was asymptomatic.
CT scan showed a focal lesion measuring $3.8 \mathrm{~cm}$ in the pancreatic tail, with clean margins, showing contrast enhancement, better appreciated on curved multiplanar reconstruction images, which was interpreted as a primary pancreas neoplasm. Physical examination was unremarkable. Laboratory values were as follows: calcium $10.3 \mathrm{mg} / \mathrm{dL}$, urinary cortisol $241.3 \mu \mathrm{g} / 24 \mathrm{~h}$, calcitonin $32.9 \mathrm{pg} / \mathrm{mL}$, and gastrin $114 \mathrm{pg} / \mathrm{mL}$. An additional ${ }^{111}$ In Octreoscan showed increased drug intake in the left abdominal quadrants.

Surgery confirmed the presence of a $4 \mathrm{~cm}$ mass in the pancreatic tail, tenaciously adherent to the retroperitoneal cavity. A distal pancreatectomy was performed. The surgical specimen consisted of the pancreatic tail $(7.5 \times 3.5 \times 2.8 \mathrm{~cm})$ containing a solid, dark red mass measuring $4 \times 2.5 \times 2.5 \mathrm{~cm}$. Microscopic examination revealed a typical splenic parenchymal pattern; an arterial vessel with partial thrombosis and four lymph nodes without morphological alterations were visible in the peripancreatic tissue.

\section{Case report 3}

A 79-year-old man with hypertension and chronic renal failure underwent an ultrasound (US) scan for further evaluation of increased prostate-specific antigen levels. Baseline US showed a left renal mass.

A contrast-enhanced CT scan confirmed the left renal mass and a distal pancreatic mass was discovered whose enhancement pattern, similar to that of the spleen, suggested an IPAS. MRI revealed a spleen-like signal intensity (SI) both on T1-weighted and T2-weighted as well as on diffusionweighted (DW) images, thus confirming the diagnosis.

The patient consequently underwent left nephrectomy, and microscopic examination of the specimen revealed a renal oncocytoma. The patient is still under radiologic follow-up, and the pancreatic mass has not shown any evolution.

\section{Case report 4}

The tail of the pancreas of a 70-year-old man showed a nodular lesion on baseline US. The patient was asymptomatic and laboratory values were unremarkable. An additional MRI confirmed the presence of an ovoid mass in the pancreatic tail whose SI and enhancement pattern were remarkably similar to those of the spleen. The patient is still undergoing clinical and radiologic monitoring.

\section{Case report 5}

A 50-year-old woman was referred to our clinic for further evaluation of a hypervascular pancreatic mass incidentally found on a CT scan (Figure 2A and B). The patient had 

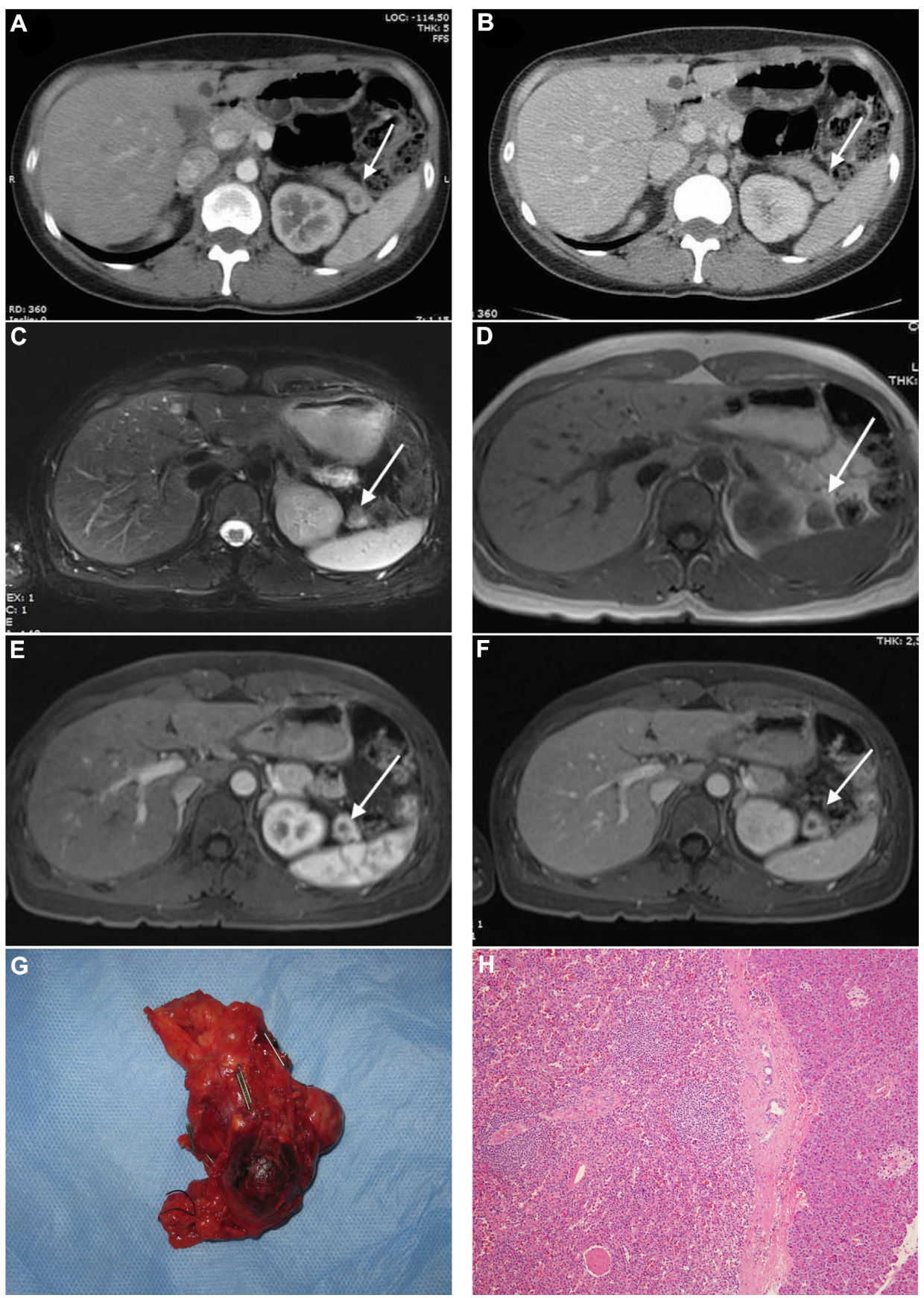

Figure I Case report I. MDCT: peripheral arterial enhancement (A) with a central hypodense area (arrow), unenhancing in both the arterial and venous phases (B). MRI: round and well-defined nodule in the pancreatic tail (arrow). The nodule shows a solid peripheral pattern, while the central hypodense area (seen on MDCT) is hypointense on T2-weighted images and isointense on TI-weighted images (C and D). Gd-enhanced MRI: solid component without significant washout (arrow) in the venous phase; arrows indicate IPAS (E and $\mathbf{F})$; the surgical specimen contains a smooth, dark red nodule $(\mathbf{G})$. Microscopic examination shows typical splenic parenchyma (magnification $\times$ I0) $(\mathbf{H})$. Abbreviations: Gd, gadolinium; IPAS; intrapancreatic accessory spleen; MDCT, multidetector-row computed tomography; MRI, magnetic resonance imaging.

previously undergone some surgical procedures for a pituitary gland adenoma.

MRI scan, performed in our institution, showed an SI pattern similar to the spleen, on T2-weighted (Figure 2C) and DW images (Figure 2D-F), as well as on Gd-enhanced images (Figure 2G). MRI was completed with gradient echo sequences before and after (Figure 2H and I) superparamagnetic iron oxide (SPIO) administration; this T2-weighted contrast agent is "captured" by the reticuloendothelial system within the liver and spleen parenchyma, thus causing a considerable signal dropout on T2-weighted images. The mass showed a significant SI decrease due to the uptake of the contrast agent (Figure 2I). A further ${ }^{111}$ In Octreoscan did not show any pathological drug uptake. The patient is still undergoing radiologic monitoring. 

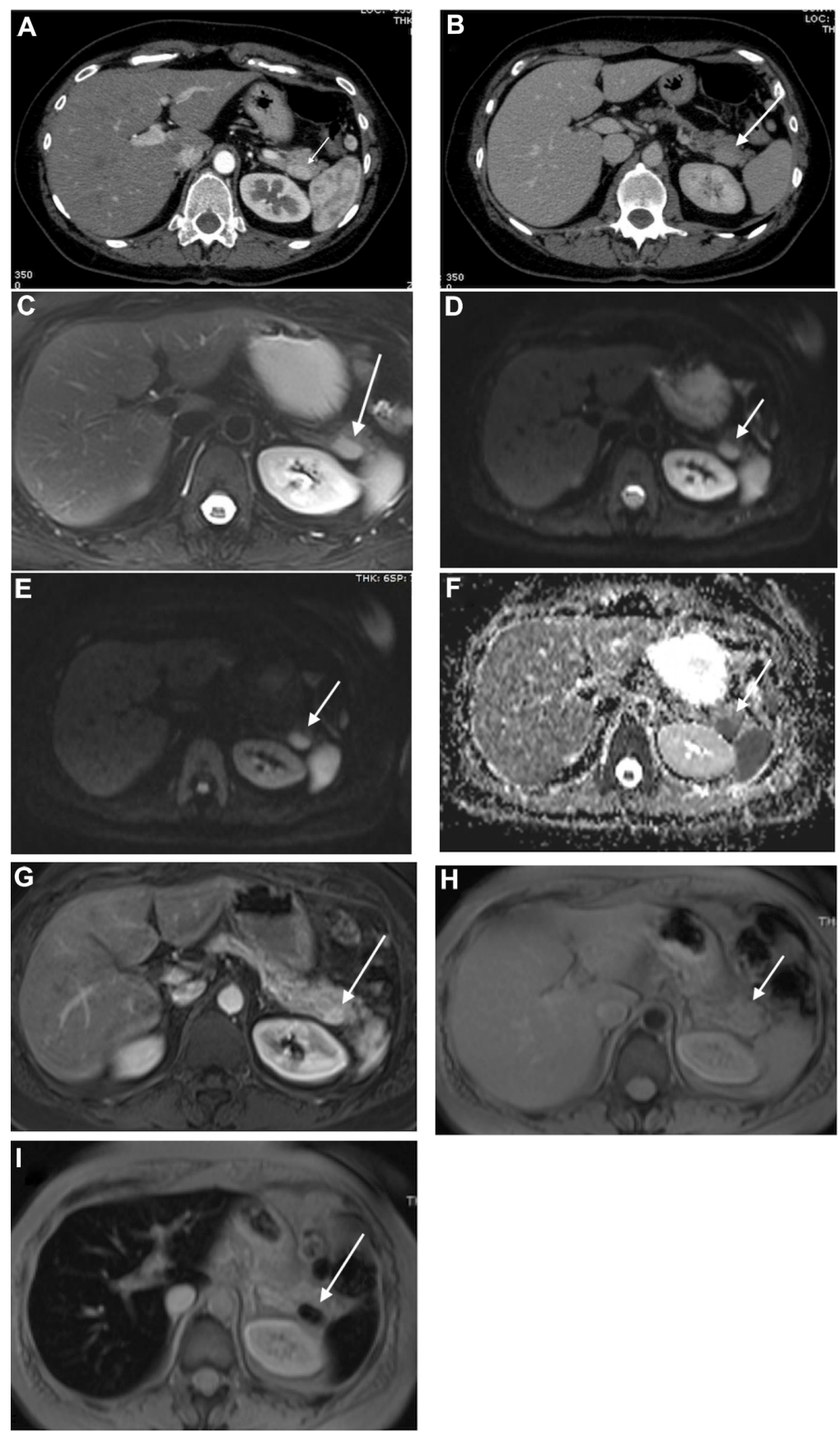

Figure 2 Case report 2. CT: hypervascular pancreatic mass (arrow) (A and B). MRI: spleen-like SI (arrow), on T2-weighted (C) and DW images (D-F) and on Gd-enhanced images (G). MRI is completed with GRE sequences before and after ( $\mathbf{H}$ and $\mathbf{I})$. SPIO administration: the mass in the tail of the pancreas (arrow) shows a significant decrease of SI due to the uptake of the contrast agent (I). Arrows indicate IPAS.

Abbreviations: CT, computed tomography; IPAS, intrapancreatic accessory spleen; MRI, magnetic resonance imaging; DW, diffusion weighted; Gd, gadolinium; GRE, gradient echo; SPIO, superparamagnetic iron oxide; SI, signal intensity.

\section{Discussion}

Accessory spleen is a relatively frequent finding, with a worldwide prevalence of $10 \%-30 \% \cdot{ }^{1-4}$ Generally, accessory spleen is located at the level of the hilum of the spleen, thus making diagnosis quite easy. However, intrapancreatic location can raise difficulties in the differential diagnosis. In this case, misdiagnosis is frequent due to its radiologic features (CT, MRI, and US), which are similar to those of hypervascular pancreatic tumors. ${ }^{5,6}$

\section{Diagnosis}

On a gray-scale baseline US examination, IPAS is usually seen as a round, oval mass with a mildly echogenic and homogeneous texture, often surrounded by a high-amplitude interface. 
In order to enhance the diagnostic power of US, Ota and $\mathrm{Ono}^{5}$ propose contrast-enhanced ultrasonography with Levovist, an US contrast agent made of microbubbles that are almost exclusively trapped by the hepatic and splenic parenchyma.

In the arterial phase, there is a typical inhomogeneous enhancement related to the different flow rate through the splenic cords, while in the portal and venous phase, IPAS shows dense, persistent enhancement, lasting from 3 minutes to 5 minutes. ${ }^{7,8}$ On CT scan, IPAS may demonstrate an inhomogeneous enhancement in the early phase, which may be a diagnostic clue. Furthermore, attenuation of IPAS in all the dynamic CT phases is usually similar to that of the spleen.

Gd-enhanced MRI might show a typical inhomogeneous enhancement in the arterial phase. ${ }^{8}$ Herédia et $\mathrm{al}^{9}$ reported their experience with dynamic Gd-enhanced MRI in five cases and stated that the discovery of a well-marginated, rounded mass in the distal tract of the tail of the pancreas with SI features of the spleen on all the precontrast and post Gd sequences suggests the diagnosis of IPAS.

SPIO MRI has been proposed as a diagnostic method since this contrast agent is targeted in the reticuloendothelial cells within the lesions. ${ }^{7,9} \mathrm{Kim}$ et $\mathrm{al}^{10}$ highlight the role of SPIO MRI in differential diagnosis based on a case series of seven patients with pancreatic masses of uncertain origin, describing an SI dropout degree quite similar to that of the spleen on SPIO-enhanced, T2-weighted images.

However, nowadays, SPIO agents have been withdrawn from the market in most countries, making it difficult to use them. DW MRI is a useful tool to distinguish IPAS from small pNETs. In fact, IPAS shows a more restricted diffusion rate on apparent diffusion coefficient maps. ${ }^{11,12}$

${ }^{111}$ In Octreoscan is highly sensitive (70\%-95\%) in detecting pNETs, and it is often used to obtain correct diagnoses. Unfortunately, normal splenic tissue shows a physiological uptake of ${ }^{111} \mathrm{In}$, thus giving rise to false-positive results. ${ }^{13}$

A proper way to distinguish IPAS from $\mathrm{pNET}$ is to submit patients to technetium (Tc) $99 \mathrm{~m}$-sulfur colloid scintigraphy or to scintigraphy with Tc99m heat-damaged red blood cells.

These tracers show specific uptake in normal liver and spleen, making the focal uptake in the pancreatic tail suggest the presence of ectopic splenic tissue. ${ }^{14}$ However, these investigations are often limited by their low resolution power in the case of small lesions.

Schwartz et $\mathrm{al}^{15}$ also suggest that radionuclide imaging with either Tc99m-sulfur colloid or Tc99m heat-damaged red blood cells should be routinely used in evaluating pancreatic masses appearing as nonfunctioning pNETs.
Schreiner et $\mathrm{al}^{16}$ report three cases reliably diagnosed by endoscopic US-guided fine-needle aspiration biopsy, which issued benign prognosis and prevented unnecessary surgery, with adequate reliability for both patients and physicians. Thus, the only safe diagnostic method appears to be direct sampling, although we must bear in mind that, despite being minimally invasive, this method could still lead to misdiagnosis due to indiscriminate collection of pancreatic islet cells. ${ }^{17}$

\section{Differential diagnosis}

\section{Pancreatic neuroendocrine tumors}

According to Jang et al, ${ }^{12}$ IPAS shows isointensity more frequently than small pNETs on DW MRI T2-weighted images. On CT scan, a pNET would be expected to enhance more uniformly than IPAS, even early in the arterial phase. ${ }^{18}$ Moreover, pNETs are frequently located within the head of the pancreas and often $(15 \%-53 \%)$ become symptomatic due to secretion of biologically active hormones. ${ }^{19}$

\section{Metastases}

Primary malignancies that commonly metastasize to the pancreas include lung, breast, gastrointestinal, and renal lesions and melanoma, lymphoma, and osteosarcoma. Imaging features that raise the suspicion of metastatic disease are multiplicity, hypervascularity, and features consistent with the primary tumor. ${ }^{20}$

\section{Solid pseudopapillary tumors}

Solid pseudopapillary tumors are rare neoplasms of the pancreas. They show homogeneous hypoechogenicity on baseline US, but contrast-enhanced ultrasonography shows peripheral rim hyperenhancement and cystic areas in the tumor, which significantly improve the proper diagnosis. ${ }^{21}$

\section{Pancreatic ductal adenocarcinoma}

On CT, larger IPASs are homogeneously enhancing and mostly mimic pNETs, but smaller lesions measuring $<1 \mathrm{~cm}$ can have a lower or heterogeneous attenuation and can mimic pancreatic ductal adenocarcinoma. ${ }^{22}$

\section{Conclusion}

Focusing on our two cases of mistaken diagnosis, in the first one, we had CT and MRI evidence of a pancreatic mass that was suspected to be a pNET due to its radiological pattern, with an unusual central hypodense core, rarely observed in IPAS. Taking into account the patients' young age, her previous malignancy diagnosis, and her desire to have the 
mass removed, we eventually went on to perform surgery, which proved to be unnecessary.

In the second case, a false-positive ${ }^{111}$ In Octreoscan led to wrong diagnosis; once again, surgery was unavoidable. Since modern radiologic techniques will probably make IPAS easier to diagnose, we believe that we should resort to any noninvasive means to avoid unnecessary surgery. We suggest that a multidisciplinary approach should guide the diagnosis, together with careful literature evaluation and appropriate judgment. When a pancreatic mass with specific features (round, ovoid, well defined) is detected by CT or MRI, an IPAS should be suspected and the case should be carefully analyzed. In our experience, DW MRI is the most reliable and accessible imaging method to achieve the correct diagnosis.

\section{Disclosure}

The authors report no conflicts of interest in this work.

\section{References}

1. Kurmann A, Michel JM, Stauffer E, Egger B. Intrapancreatic accessory spleen misdiagnosed as a non secreting endocrine tumor: case report and review of the literature. Case Rep Gastroenterol. 2010;4:210-214.

2. Meyer-Rochow GY, Gifford AJ, Samra JS. Intrapancreatic splenunculus. Am J Surg. 2007;194:75-76.

3. Kavic MS, Park A. Intrapancreatic accessory spleen: deficiency in diagnosis or therapeutic success? J Gastrointest Surg. 2009;13:396.

4. Marcin Z, Zembala-Nozynska E, Sczasny J, Straczynski M, Widel M. Intrapancreatic accessory spleen imitating a pancreatic neoplasm. Pol Przegl Chir. 2011;83(10):568-570.

5. Ota T, Ono $\mathrm{S}$. Intrapancreatic accessory spleen: diagnosis using contrast enhanced ultrasound. Br J Radiol. 2004;77:148-149.

6. Suriano S, Ceriani L, Gertsch P, Crippa S, Giovanella L. Accessory spleen mimicking a pancreatic neuroendocrine tumor. Tumori. 2011;97(6): $39 \mathrm{e}-41 \mathrm{e}$.

7. Kim SH, Lee JM, Han JK, et al. Intrapancreatic accessory spleen: findings on MR Imaging, CT, US and scintigraphy, and the pathological analysis. Korean J Radiol. 2008;9(2):162-174.

8. Kim SH, Lee JM, Lee JY, Han JK, Choi BI. Contrast enhanced sonography of intrapancreatic accessory spleen in six patients. Am J Roengtenol. 2007;188:422-428.
9. Herédia V, Altun E, Bilaj F, Ramalho M, Hyslop BW, Semelka RC. Gadolinium- and superparamagnetic-iron-oxide-enhanced MR findings of intrapancreatic accessory spleen in five patients. Magn Reson Imaging. 2008;26:1273-1278.

10. Kim SH, Lee JM, Han JK, et al. MDCT and superparamagnetic iron oxide (SPIO)-enhanced MR findings of intrapancreatic accessory spleen in seven patients. Eur Radiol. 2006;16:1887-1897.

11. Kang BK, Kim JH, Byun JH, et al. Diffusion-weighted MRI: usefulness for differentiating intrapancreatic accessory spleen and small hypervascular neuroendocrine tumor of the pancreas. Acta Radiol. 2014;55(10):1157-1165.

12. Jang KM, Kim SH, Lee SJ, Park MJ, Lee MH, Choi D. Differentiation of an intrapancreatic accessory spleen from a small $(<3-\mathrm{cm})$ solid pancreatic tumor: value of diffusion-weighted MR imaging. Radiology. 2013;266(1):159-167.

13. Lebtahi R, Cadiot G, Marmuse JP, et al. False-positive somatostatin receptor scintigraphy due to an accessory spleen. J Nucl Med. 1997;38: 1979-1981.

14. Brasca LE, Zanello A, De Gaspari A, et al. Intrapancreatic accessory spleen mimicking a neuroendocrine tumor: magnetic resonance findings and possible diagnostic role of different nuclear medicine tests. Eur Radiol. 2004;14:1322-1323.

15. Schwartz TL, Sterkel BB, Meyer-Rochow GY, et al. Accessory spleen masquerading as a pancreatic neoplasm. Am J Surg. 2009;197:61-63.

16. Schreiner AM, Mansoor A, Faigle DO, Morgan TK. Intrapancreatic accessory spleen: mimic of pancreatic endocrine tumor diagnosed by endoscopic ultrasound-guided fine-needle aspiration biopsy. Diagn Cytopathol. 2008;36:262-265.

17. Arkadopoulos N, Athanasopolous P, Stafyla V, et al. Intrapancreatic accessory spleen issues: diagnostic and therapeutic challenges. $J$ Pancreas. 2009;10(4):400-405.

18. Sothilingam N, Leedahl T, Kriegler S, Kanthan R, Moser MA. Intrapancreatic accessory spleen: a case report and review of the literature. Int J Surg Case Rep. 2011;2(6):128-130.

19. Dralle H, Krohn SL, Karges W, Boehm BO, Brauckhoff M, Gimm O. Surgery of resectable nonfunctioning neuroendocrine pancreatic tumors. World J Surg. 2004;28:1248-1260.

20. To'o KJ, Raman SS, Yu NC, et al. Pancreatic and peripancreatic diseases mimicking primary pancreatic neoplasia. Radiographics. 2005;25: 949-965.

21. Jiang L, Cui L, Wang J, Chen W, Miao L, Jia L. Solid pseudopapillary tumors of the pancreas: findings from routine screening sonographic examination and the value of contrast-enhanced ultrasound. J Clin Ultrasound. 2015;43(5):277-282.

22. Al-Hawary MM, Kaza RK, Azar SF, Ruma JA, Francis IR. Mimics of pancreatic ductal adenocarcinoma. Cancer Imaging. 2013;13(3): 342-349.
Open Access Surgery

\section{Publish your work in this journal}

Open Access Surgery is an international, peer-reviewed, open access journal that focuses on all aspects of surgical procedures and interventions. Patient care around the peri-operative period and patient outcomes post surgery are key topics. All grades of surgery from minor cosmetic interventions to major surgical procedures are covered. Novel techniques Submit your manuscript here: http://www.dovepress.com/open-access-surgery-journal

\section{Dovepress}

and the utilization of new instruments and materials, including implants and prostheses that optimize outcomes constitute major areas of interest. The manuscript management system is completely online and includes a very quick and fair peer-review system. Visit http://www.dovepress.com/ testimonials.php to read real quotes from published authors. 